\title{
Boric acid catalyzed one-pot synthesis of $[1,2,4]$ triazolo quinazolinone Derivatives
}

\author{
Kabeer A. Shaikh ${ }^{\mathrm{a}^{*}}$, Sagar R. Kande ${ }^{\mathrm{b},}$ C. B. Khillare ${ }^{\mathrm{c}}$ \\ ${ }^{a *}$ P.G. Department of chemistry, Sir Sayyed College of Arts, Commerce and Science, Aurangabad, \\ Maharashtra, India \\ ${ }^{b}$ P.G. Department of chemistry, New Arts, Commerce and Science College, Shevgaon, Ahmednagar, \\ Maharashtra, India \\ ${ }^{c}$ P.G. Department of Chemistry, Maulana Azad College, Aurangabad- (M.S.) India
}

\begin{abstract}
We present a rapid, efficient one pot synthesis of [1,2,4]triazolo [5,1-b]quinazolin-8(4H)-one derivatives by condensation of 3-amino-1,2,4-triazole with aldehydes and dimedone in the presence of boric acid (5 mmol\%) as a catalyst. This protocol corresponds in short reaction time, low cost, high reaction yield, no need of chromatographic separation with green aspects by using water soluble catalysts.
\end{abstract}

Keywords: aldehyde, 3-amino-1,2,4-triazole, dimedone, boric acid.

\section{Introduction}

Multicomponent reactions(MCRs) in which three or more reactants are combined to form a product containing substantial elements of all reactants ${ }^{1,2}$. One pot MCR process have an efficient and powerful tool for the construction of complex molecules ${ }^{3,4}$. One pot MCR provides many chemical reactions which attributes such as minimization of use of silica gel for chromatographic purification of intermediate compounds, use of solvent $^{5}$. MCR's have been exploited as a powerful tool for the assembly of large library of biologically active molecules ${ }^{6}$.Nitrogen containing heterocyclic, triazolo quinazolinone ring system exhibit biological activities such as antihypertensive ${ }^{7}$, antihistamic ${ }^{8}$, analgesic, anti-inflammatory ${ }^{9}$, anticancer ${ }^{10}$ and anti-HIV activities ${ }^{11 a}$. More over many naturally occurring and synthetic compounds containing triazole derivatives scaffold also posses interesting diverse pharmaceutical, agrochemical properties. Some important drugs which are present in market such as terconazole, alperazolame, triazolame and rizatriptan ${ }^{11 \mathrm{~b}}$ (figure 1)contains 1,2,4 Triazole nucleus. A three component condensation of a substituted aldehyde, dimdone and 3-amino-1,2,4-triazole as an amine source is well known. An important existing procedure for the synthesis of 1,2,4-triazolo[5,1-b]quinolin$8(4 \mathrm{H})$-one derivatives have been carried out by refluxing starting compounds in $\mathrm{DMF}^{12 a, \mathrm{~b}}$, using sulphamic acid in acetonitrile as a solvent and reflux condition ${ }^{13}$, ionic liquids ${ }^{14}$, hetero polyacids ${ }^{15}$, molecular iodine ${ }^{16}$.

However some of these methodologies have many limitations of harsh reaction condition, use of expensive catalysts, long reaction time and tedious work up, low yield. Hence the development of rapid, simple, efficient, clean, high yielding, environmentally benign protocol using new catalyst for the synthesis of this compound is desirable. Boric acid is a water soluble inexpensive, stable, readily available, easy to handle catalyst and has been found effective in various transformations such as synthesis of aza Michael ${ }^{17}$, thia Michael $^{18}$, transesterification of ethylacetoacetate ${ }^{19}$, synthesis of $\alpha$-hydroxyamides ${ }^{20}$, bromination, oxidation of sulphides $^{21}$, beginelli reaction ${ }^{21}, 1,5$ - benzodiazepine derivatives ${ }^{22}$, 2-amino,3-5 dicarbonitrile-6-thio-pyridene ${ }^{23}$. It is therefore important to examine the behavior of boric acid as a catalyst in the synthesis of 1,2,4-triazolo quinolinone derivatives.

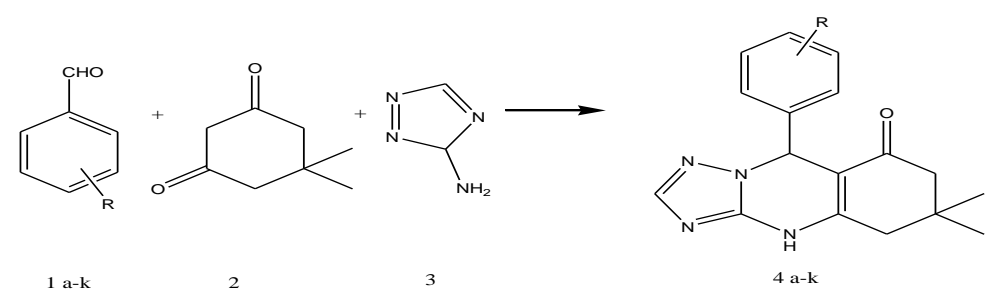

Scheme : Synthesis of 1,2,4-triazolo[5,1-b]quinolin-8(4H)-one derivatives

To determine the optimum concentration of the boric acid catalyst, we investigated model reaction at it's different concentrations. The product was formed in $91.2 \%, 98.2 \%, 96.1 \%, 89.7 \%$ and $83.5 \%$ (Table 1) 
yield by using concentration of boric acid catalyst i.e. 3, 5, 10, 15, 20 mmol\% respectively. These result shows $5 \mathrm{~mol} \%$ of boric acid is sufficient to carry out the reaction smoothly. Herein we report a synthesis of $1,2,4-$ triazolo[5,1-b]quinolin-8(4H)-one derivatives.

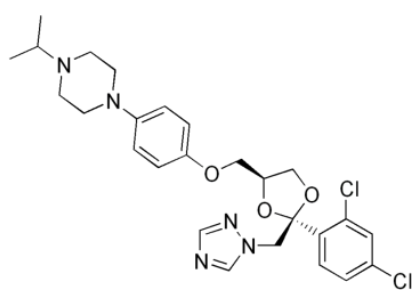

Terconazole<smiles>Cc1nnc2n1-c1ccc(Cl)cc1C(c1ccccc1)=NC2</smiles>

Alprazolam<smiles>Cc1nnc2n1-c1ccc(Cl)cc1C(c1ccccc1Cl)=NC2</smiles>

Triazolam<smiles>CN(C)CCc1c[nH]c2ccc(Cn3cncn3)cc12</smiles>

Rizatriptan

Figure 1

\section{Experimental}

All solvents and chemicals which are used in this reaction are of commercial grade quality without any purification and were produced from Merck(Germany). Silica gel coated aluminium sheets (merck product) were used for thin layer chromatography to monitor progress of the reaction. Melting points were measured by an open capillary tube method and are uncorrected. ${ }^{1} \mathrm{H}$ and ${ }^{13} \mathrm{C}$ NMR were carried out on a SpectronicGenesys 2 Spectrophotometer $300 \mathrm{MHz}$ in DMSO-d6 solvent. IR spectra recorded from $\mathrm{KBr}$ disk in the range of 4000$200 \mathrm{~cm}^{-1}$ on the Shimadzu FTIR spectrometer (Model no. 8400). MASS spectra were obtained on Polaris-Q Thermospecific GC-MS.

\section{Typical procedure for the synthesis of [1,2,4]triazolo [5,1-b]quinazolin- 8(4H)-one derivatives.}

The mixture of 3-amino-1,2,4-triazole 3 ( $1 \mathrm{mmol})$, p-nitro benzaldehyde (1mmol), dimedone 2 (1mmol) and $5 \mathrm{mmol} \%$ boric acid was taken in an agate mortar and pestle set and ground it for 2 minutes. Then $5 \mathrm{ml}$ ethyl acetate was added to the mixture. After evaporation of the solvent product was separated out and washed with water then dried at low pressure to obtain the crude product. Completion of the reaction was monitored by TLC. The product was purified by recrystallization by using ethyl alcohol as a solvent to get expected product $4 \mathrm{a}-\mathrm{k}$. All the compounds were synthesized and characterized using their data in literature.

Table 1 Effect of the catalyst concentration ${ }^{\text {a }}$

Entry Boric Acid (mmol\%) $\quad$ Yield $^{\mathrm{b}}$

\begin{tabular}{lcc}
\hline 1 & 3 & 91 \\
$\mathbf{2}$ & $\mathbf{5}$ & $\mathbf{9 8}$ \\
3 & 10 & 96 \\
4 & 15 & 89 \\
5 & 20 & 83 \\
\hline
\end{tabular}

Note: Bold values indicate optimized condition

${ }^{a}$ Reaction condition: $\mathbf{1 a}(1 \mathrm{mmol}), \mathbf{2}(1 \mathrm{mmol})$ and $\mathbf{3}(1 \mathrm{mmol})$ ground in agate mortar, $\mathrm{rt}$ ${ }^{\mathrm{b}}$ Isolated yield.

Table 2: Boric acid catalyzed synthesis of Triazolo quinazolinones-

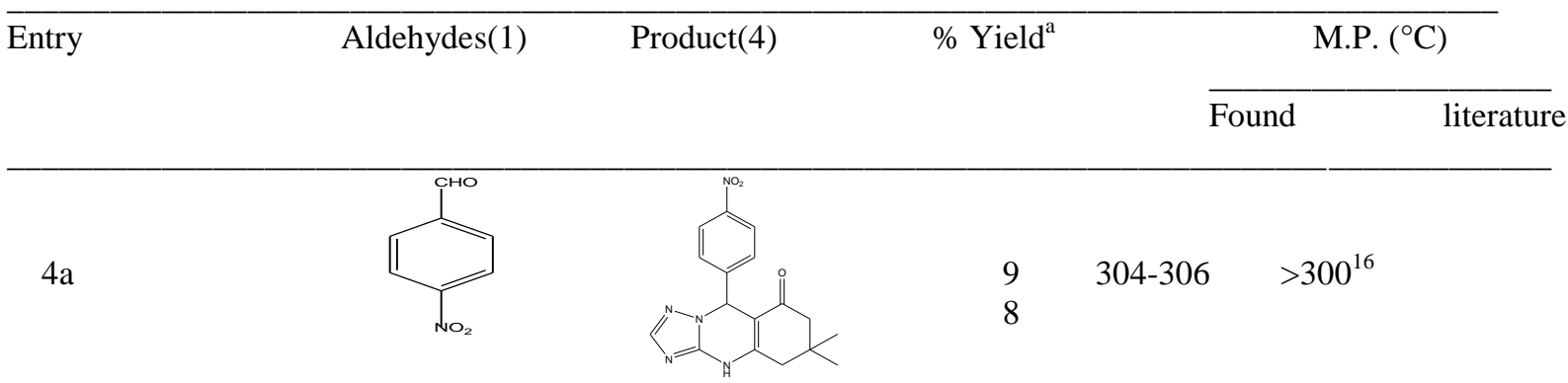


$4 \mathrm{~b}$<smiles>Cc1ccc(O)cc1</smiles>

$4 \mathrm{c}$<smiles>COc1ccc(O)cc1</smiles>

$4 d$

$4 \mathrm{e}$

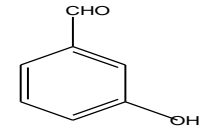<smiles>Cc1ccc(O)cc1O</smiles><smiles>Cc1ccccc1O</smiles>

$4 \mathrm{f}$

$4 \mathrm{~g}$<smiles>COc1ccccc1</smiles>

$4 \mathrm{~h}$<smiles>CCc1cc(O)ccc1O</smiles><smiles>Cc1cccc(O)c1</smiles>

$4 \mathrm{i}$

$4 \mathrm{j}$<smiles>CC(C)c1ccc(O)cc1</smiles><smiles>CCC1CCC(C)CC1</smiles>

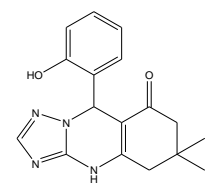<smiles>O=C1CC2(CC2)CC2CC3CCC(C3)CC12</smiles>

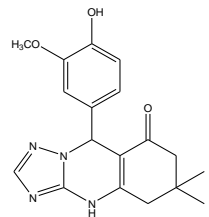

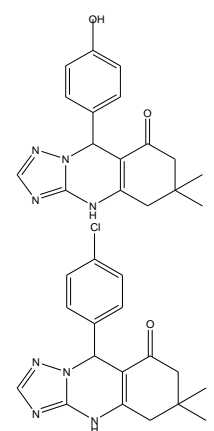

$90>300$

$>300^{16}$

$96 \quad 303-305 \quad 304-306^{16}$

$85 \quad 291-293 \quad 289-290^{16}$

$82 \quad 301-303$

$83 \quad 294-296 \quad--$

$83 \quad 249-251 \quad 250-252^{16}$

$91 \quad 287-290 \quad---$

$94 \quad 272-275 \quad 290-293^{14}$

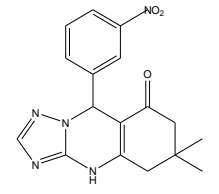

$93 \quad 288-291 \quad 285-287^{14}$

92

227- $228-230^{16}$ 229

2.1. Spectroscopic Data for representative and newly synthesized compounds is listed below: 
6,6-Dimethyl-9-(4-nitro-phenyl)-5,6,7,9-tetrahydro-4H- 1,2,4-triazolo[5,1-b]quinazolin-8-one (4a): Pale yellow solid, Yield- $98.2 \%$, M.P.=304-306 C, IR- 2962, 1645, 1568, 1354, 1250, $720 \mathrm{~cm}-1$; ${ }^{1} \mathrm{H}-\mathrm{NMR}$ (DMSO-d6, 300MHz): $\delta \mathrm{H} 1.02$ (s, 3H,CH3), 1.10 (s, 3H, CH3), 2.10 (d, J=16.20 Hz,2H), 2.30 (d, J=16.40 Hz, $2 \mathrm{H}), 2.55(\mathrm{~d}, \mathrm{~J}=16.20,2 \mathrm{H}), 6.35(\mathrm{~s}, 1 \mathrm{H}), 7.14(\mathrm{~d}, \mathrm{~J}=8.2 \mathrm{~Hz}, 2 \mathrm{H}), 7.61(\mathrm{~d}, \mathrm{~J}=8.2 \mathrm{~Hz}, 2 \mathrm{H}), 8.04(\mathrm{~s}, 1 \mathrm{H}), 11.03(\mathrm{~s}$, $1 \mathrm{H}, \mathrm{NH}) \mathrm{ppm} .{ }^{13} \mathrm{C} \mathrm{NMR}(75 \mathrm{MHz}, \mathrm{CDCl} 3): \delta 190.32,155.21,132.11,130.11,120.47,112.42,68.72,66.46$, 25.19, 17.21. MS m/z (ESI); $340[\mathrm{M}+\mathrm{H}]^{+}$.

6,7-dihydro-9-(2,4-dihydroxyphenyl)-6,6-dimethyl-[1,2,4]triazolo[5,1-b]quinazolin-8(4H,5H,9H)one(4e): Pale yellow solid, Yield- 82.7\% , M.P.=301-303॰C, IR- 3090, 2941, 1643, 1610, 1438, 1261,839 cm-1; ${ }^{1} \mathrm{H}-\mathrm{NMR}$ (DMSO-d6, 300MHz): $\delta \mathrm{H} 0.99$ (s, 3H, CH3), 1.09 (s, 3H, CH3), 2.08 (q, J = 14.17, 23.03 Hz, $2 \mathrm{H},-\mathrm{CH} 2), 2.49$ (s, 2H, - CH2), $6.48(\mathrm{~s}, 1 \mathrm{H},-\mathrm{CH}), 7.22-7.31(\mathrm{~m}, 2 \mathrm{H}, \mathrm{Ar}-\mathrm{H}), 7.45(\mathrm{~s}, 1 \mathrm{H}, \mathrm{Ar}-\mathrm{H}), 7.59(\mathrm{~s}, 1 \mathrm{H}$, $\mathrm{Ar}-\mathrm{H}), 10.92$ (s, 1H, NH); ${ }^{13} \mathrm{C}$ NMR $(75 \mathrm{MHz}, \mathrm{CDCl} 3): \delta 161.39,141.86,129.47,125.12,123.35,110.18$, $60.52,59.48,15.71,13.11 . \mathrm{MS} \mathrm{m} / \mathrm{z}(\mathrm{ESI}) ; 327[\mathrm{M}+\mathrm{H}]^{+}$.

6,7-dihydro-9-(2-hydroxyphenyl)-6,6-dimethyl-[1,2,4]triazolo[5,1-b]quinazolin-8(4H,5H,9H)-one (4f): Pale yellow solid, Yield- 83.7\% , M.P.=294- 296 ${ }^{\circ}$ C, IR- 3089, 2938, 1663, 1567, 1342, 1260, $788 \mathrm{~cm}-1 ;{ }^{1} \mathrm{H}-$ NMR (DMSO-d6, 300MHz): $\delta \mathrm{H} 1.02$ (s, 3H, CH3), 1.09 (s, 3H, CH3), 2.09 (q, J = 11.92, 16.89 Hz, 2H, CH2), 2.35 (s, 2H, -CH2), $6.87(\mathrm{~s}, 1 \mathrm{H},-\mathrm{CH}), 7.30-7.38$ (m, H, Ar-H), 7.22-7.31 (m, H, Ar-H), 7.11 (m, 1H, Ar$\mathrm{H}), 7.53$ (s, 1H, Ar-H), 7.31 (s, 1H, Ar-H), 11.05 (s, 1H, NH);

${ }^{13} \mathrm{C}$ NMR (75 MHz, CDCl3): $\delta 162.87,142.33,130.76,126.94,123.31,112.23,60.89,58.51,14.83,12.72 . \mathrm{MS}$ $\mathrm{m} / \mathrm{z}(\mathrm{ESI}) ; 311[\mathrm{M}+\mathrm{H}]^{+}$.

6,7-dihydro-9-(4-hydroxy-3-methoxyphenyl)-6,6-dimethyl-[1,2,4]triazolo[5,1-b]quinazolin8(4H,5H,9H)-one (4h): Pale yellow solid, Yield- 91.4\% , M.P.=287-290॰C, IR- 3081, 2968, 1655, 1589, 1375, 1242, $764 \mathrm{~cm}-1 ;{ }^{1} \mathrm{H}-\mathrm{NMR}$ (DMSO-d6, 300MHz): $\delta \mathrm{H} 1.01$ (s, 3H,CH3), 1.08 (s, 3H, CH3), 2.11 (q, J = 13.62, $22.79 \mathrm{~Hz}, 2 \mathrm{H},-\mathrm{CH} 2), 2.43$ (s, 2H, - $\mathrm{CH} 2) 3.70$ (s, 3H, -OCH3), 6.71 (s, 1H, -CH), 7.11-7.20 (m, 2H, Ar-H), 7.37 (s, 1H, Ar-H), 7.49 (s, 1H, Ar-H), 11.21 (s, 1H, NH) ppm.

${ }^{13} \mathrm{C}$ NMR $(75 \mathrm{MHz}, \mathrm{CDCl} 3): \delta 189.74,157.46,155.83,149.44,146.57,133.12,131.77,126.51,122.48,101.17$, 49.33, 48.10, 30.72, 27.56, 25.37. MS m/z (ESI); $341[\mathrm{M}+\mathrm{H}]^{+}$.

\section{Conclusion}

In conclusion we have developed an environmentally benign protocol for the synthesis of 1,2,4triazolo[5,1-b] quinolin-8(4H)-one derivatives by using 3-amino-1,2,4-triazole, benzaldehydes and dimedone in the presence of $5 \mathrm{mmol} \%$ Boric acid as a catalyst. The catalyst was found to catalyze reaction at room temperature. The advantages of this procedure are short reaction time, low cost, high reaction yield, no need of chromatographic separation use of water soluble catalyst.

\section{Acknowledgments}

The author SRK thankful to Principal Sir Sayyed College,Aurangabad, Principal, New Arts College, Shevgaon, Ahmednagar and also thankful to UGC, New Delhi for financial assistance (wide file no.-41357/2012).

\section{References}

[1] J. Zhu, H.Bienayme, (Eds.) Multicomponent Reactions, Wiley-VCH: Weinhein, Germany, 2005.

[2] A. Domling, Chem. Rev. 106, 2006, 17-89.

[3] M. Syamala, Org. Prep. Proced. Int. 41, 2009, 1-68.

[4] M. Syamala, Org. Prep. Proced. Int. 37, 2005, 103.

[5] A. Domling and I. Ugi, Angew Chem Int. Ed. 39, 2000, 3168-3210.

[6] G. Sklute and I. Marek, J.Am.Chem.Soc. 128, 2006, 4642.

[7] V. Alagarsamy, V. R. Solomon and M. Murugan, Bio. org. Med. Chem. 15, 2007, 4009-4015.

[8] V. Alagarsamy, Pharmazie 59, 2004, 3753-3755.

[9] V. Alagarsamy, G. Murugananthan and R. Venkateshperumal, Biol. Pharm. Bull. 26, 2003, 1711-1714.

[10] M. J. Hour, L. J. Huang, S. C. Kuo, Y. Xia, K. Bastow, Y. Nakanishi, E. Hamel and K. H. Lee, J. Med. Chem. 43, 2000, 44794487.

[11] (a) V. Alagarsamy, R. Revathi, S. Meena, K. V. Ramasheshu, S. Rajashekarn and De Clercq, E. Indian J. Pharm. Sci. 66, 2004, 459-462. (b) K. Zamani, K. Faghihi, T. Tofighi and M. Shariatzadeh, Turk. J. Chem. 28, 2004, 95-100.

[12] (a) V. V. Lipson, S. M. Desenko, M. G. Shirobokova and V. V. Borodina, Chem. Heterocycl. Compd. 39, 2003, 1213. (b) V. V. Lipson, S. M. Desenko, M. G. Shirobokova, O. V. Shishkin and V. D. Orlov, Chem. Heterocycl. Compd. 39, $2003,1041$.

[13] M. M. Heravi, F. Derikv and, L. Ranjbar, Synth. Commun. 40, 2010, 677-685.

[14] K. Kumari, D. S. Raghuvanshi and K. N.Sing, Org. Prep. Proced. Int. 44, 2012, 460-466.

[15] M. M. Heravi, L. Ranjbar, F. Derikvand, B. Alimadadi, H. A. Oskooie and F. F. Bamoharram, Mol. Divers. 12, 2008, 181-185.

[16] R. G. Puligoundla,; S. Karnakanti, R. Bantu, N. Kommub, S. B.; S. B. Kondra and L. Nagarapu, Tetrahedron Letters 54, 2013, 2480-2483.

[17] M. K. Chaudhuri, S. Hussain, M. L. Kantamb and B. Neelima, Tetrahedron Lett. 46, 2005, 8329.

[18] K. Chaudhuri and S. Hussain, J. Mol. Catal. A. Chem. 269, 2007, 214

[19] G. C. M. Kondaiah, L. A. Reddy, K. S. Babu, V. M. Gurav, , K. G. Huge, R. Bandichhor, P. P. Reddy, A. Bhattacharya and R. V. Anand, Tetrahedron Lett. 49, 2008, 106. 
[20] J. S. Kumar, S.C. Jonnalagadda and V. R. Mereddy, Tetrahedron Lett. 51, 2010, 779.

[21] A. Rostami and J. Akradi, Tetrahedron Lett. 51, 2010, 3501.

[22] S. Tu, F. Fang, C. Miao, H. Jiang, Y. Feng, D. Shi and X. Wang, Tetrahedron Lett. 44, 2003, 6153.

[23] X. Zhou, M. Y. Zhang, S. T. Gao, J. J. Ma, C. Wang and C. Liu, Chin. Chem. Lett. 20, 2009, 905.

[24] P. V. Shinde, S. S. Sonar, B. B. Shingate and M. S. Shingare, Tetrahedron Lett. 51, 2010, 1309. 